\title{
Kinematic changes in patients with severe knee osteoarthritis are a result of reduced walking speed rather than disease severity
}

Petros Ismailidis ${ }^{1,2,3}$ Christian Egloff ${ }^{1,}$ Lea Hegglin ${ }^{1,4}$, Geert Pagenstert ${ }^{2,5}$ Rolf Kernen ${ }^{6}$, Eckardt Anke $^{7}$, Ilchmann Thomas ${ }^{7}$, Annegret Mündermann ${ }^{1,2,3,8}$, Corina Nüesch ${ }^{1,2,3,8}$

${ }^{1}$ Department of Orthopaedics and Traumatology, University Hospital Basel, Basel, Switzerland

${ }^{2}$ Department of Clinical Research, University of Basel, Basel, Switzerland ${ }^{3}$ Department of Biomedical Engineering, University of Basel, Basel, Switzerland ${ }^{4}$ Institute of Physiotherapy, Zurich University of Applied Sciences, Winterthur, Switzerland

${ }^{5}$ Clinic for Orthopaedics Clarahof, Clarahofweg 19a, 4058 Basel, Switzerland ${ }^{6}$ Clinic for Orthopaedics Claraortho, Claragraben 82, 4058 Basel, Switzerland ${ }^{7}$ Hirslanden Klinik Birshof , Reinacherstrasse 28, , 4142 Münchenstein, Switzerland ${ }^{8}$ Department of Spine Surgery, University Hospital Basel, Basel, Switzerland

\section{Original Research Article}

June 2020

Author Version - accepted for publication in Gait and Posture

doi: https://doi.org/10.1016/j.gaitpost.2020.05.008

Address of correspondence: Dr. Petros Ismailidis

Department of Orthopaedics and Traumatology

University Hospital Basel

Spitalstrasse 21

4031 Basel, Switzerland

Tel. +41613287133

Email petrosismailidis@gmail.com

Acknowledgements: Funding was received from the Department of Orthopaedics of the University Hospital Basel, the Foundation for Funding Science and Education at the Department of Surgery at the University of Basel, Swiss Orthopaedics, Merian Iselin Foundation and Deutsche Arthrose-Hilfe e.V. The funding sources had no involvement in any aspect of the study. 


\section{Abstract}

Background: Kinematic changes in patients with knee osteoarthritis (OA) have been extensively studied. Concerns have been raised whether the measured spatiotemporal and kinematic alterations are associated with disease progression or merely a result of reduced walking speed.

Research question: The purpose of this study was to investigate the effect of walking speed on kinematic parameters in patients with knee OA using statistical parametric mapping (SPM).

Methods: Twenty-three patients with unilateral knee OA scheduled for a total knee replacement and 28 age matched control subjects were included in this study. Spatiotemporal parameters and sagittal plane kinematics were measured in the hip, knee, and ankle using the inertial sensors system RehaGait ${ }^{\circledR}$ while walking at a self-selected normal (patients and controls) and slow walking speed (controls) for a distance of 20 meters. Gait parameters were compared between groups for self-selected walking speed and for matched walking speed using SPM with independent sample $\mathrm{t}$ tests.

Results: At self-selected walking speed, patients had significantly lower knee flexion during stance (maximum difference, $\left.-6.8^{\circ}\right)$ and during swing $\left(-11.0^{\circ}\right)$, as well as higher ankle dorsiflexion during stance phase $\left(+12.5^{\circ}\right)$ and lower peak hip extension at the end of stance compared to controls $\left(+4 \cdot 2^{\circ}\right)$. At matched speed, there were no significant differences in joint kinematics between groups.

Significance: Differences in sagittal plane gait kinematics between patients with knee OA and asymptomatic controls appear to be mainly a result of reduced walking speed. These results emphasize the importance of considering walking speed in research on gait kinematics in patients with knee $\mathrm{OA}$ and in clinical trials using gait parameters as outcome measures.

Key words: Kinematics; walking speed; knee osteoarthritis; gait analysis; inertial sensors 


\section{Introduction}

Spatiotemporal and kinematic differences during walking between patients with knee osteoarthritis (OA) and asymptomatic control groups have been extensively studied with marker- and camera-based gait analysis systems [1] and more recently with inertial sensor systems [2-4]. For instance, patients with knee OA typically walk at slower walking speed and with shorter stride length $[5,6]$, lower cadence $[6-9]$ and longer stride duration $[5,7,8]$ than asymptomatic groups. Moreover, patients with knee OA have been reported to have greater peak knee flexion at initial contact [10-12] and lower knee flexion during stance and swing phase $[5,13]$ than controls. In addition, observed differences in adjacent joints in patients with severe knee OA include smaller ankle range of motion (ROM) and lower peak hip extension [5].

Although spatiotemporal and kinematic changes in patients with knee OA have been documented and associated with different stages of OA progression [1], in most of these studies gait analyses were performed while patients and control subjects walked at their respective personal self-selected speed. However, walking speed is known to influence gait parameters in healthy individuals [14]. Therefore, concerns have been raised that the observed alterations only to a small degree result from the disease progression but are rather the effect of the reduced self-selected walking speed [15]. For instance, some authors used statistical methods such as analysis of covariance to elucidate the influence of speed on differences in kinematic parameters between patient and control groups [16, 17]. However, Astephen Wilson [15] judged this method as statistically inappropriate and misleading, and called for experimental ways to control speed.

In recent years, inertial sensors have become increasingly popular for measuring gait mechanics with 14 systems being reported in 24 studies in the recent literature [18]. Inertial sensor systems are easy to use, do not require a laboratory and have been validated and shown to be able to detect gait alterations reliably [19-21] compared to traditional gait analysis systems 
using reflecting markers and high speed cameras in a gait laboratory. Several successful applications of inertial sensor systems for measuring gait in patients with knee OA have been recently reported [2-4]. Statistical parametric mapping (SPM) is an appropriate method for examining 1-dimensional data such as kinematic parameters collected from inertial sensor systems and provides the advantage of analyzing entire time series contrary to an approach comparing isolated values $[22,23]$. SPM has also been used to compare knee kinematics in patients after rupture of the anterior cruciate ligament to those in asymptomatic individuals [24]. The purpose of this study was to experimentally determine the effect of walking speed on differences in kinematic parameters measured with an inertial sensor system between patients with knee OA and asymptomatic control subjects using SPM.

\section{Methods}

\subsection{Participants}

Twenty-three patients with unilateral knee OA (13 right knees; 11 female; mean (standard deviation (SD)), age: 66.1 (8.9) years; height: $1.68(0.11) \mathrm{m}$; body mass: 80.6 (15.5) $\mathrm{kg}$; body mass index (BMI): $28.1(3.8) \mathrm{kg} / \mathrm{m}^{2}$ ) and 28 age matched control subjects (18 female; age: 68.8 (6.5) years; height: 1.67 (0.09) m; body mass: 70.2 (12.3) kg; BMI: 24.9 (3.8) kg/m²) were enrolled in this study. Patients were recruited from our hospital and from local orthopaedic clinics. Inclusion criteria for patients were: age $\geq 30$ years; diagnosed unilateral OA of the knee; and scheduled for total knee arthroplasty (TKA). Asymptomatic control subjects were recruited from the local community via online advertisement on our web page and via word of mouth advertisement. Inclusion criteria for the asymptomatic control subjects were: age $\geq 30$ years; no clinical diagnosis of OA, rheumatoid arthritis or history of knee or hip trauma or pain at the time of the measurement. Exclusion criteria were: Knee Osteoarthritis Outcome Score (KOOS) 
$<90$ in the pain subcategory. Further exclusion criteria for both groups were: BMI $>35 \mathrm{~kg} / \mathrm{m}^{2}$; use of walking aids; neuromuscular disorders affecting gait; and inability to follow procedures due to psychological disorders or dementia. All subjects gave written informed consent prior to participation. This study was approved by the regional review board and conducted in accordance with the Declaration of Helsinki.

\subsection{Clinical evaluation}

The Kellgren/Lawrence $(\mathrm{K} / \mathrm{L})$ radiographic severity grade for the affected knee of the patients was determined based on anterior-posterior and lateral standing views of the knee [25]. The subjective severity of the OA was assessed using the Knee Osteoarthritis Outcome Score (KOOS) [26]. The combination of a K/L Grade 3 or 4 and symptoms leading to the decision to perform a TKA was defined as severe knee OA [5].

\subsection{Gait analysis}

Gait analyses were performed using the inertial sensor system RehaGait ${ }^{\text {(Hasomed, }}$ Magdeburg, Germany). RehaGait ${ }^{\circledR}$ consists of seven inertial sensors that are placed bilaterally at the lateral foot, lateral lower leg and lateral thigh as well as on the pelvis dorsal at the height of the $5^{\text {th }}$ lumbar vertebra (Fig. 1). Participants first walked for a distance of 20 meters in a flat, well-lit hallway at their normal self-selected speed. In addition, control subjects were asked to complete a second trial while walking at a slow self-selected speed in an attempt to capture spatiotemporal and kinematic parameters at a matched speed.

The spatiotemporal parameters (walking speed, cadence, stride length and stride duration) and sagittal plane joint kinematics of the ankle, knee and hip were computed by the manufacturer's software (Hasomed, Magdeburg, Germany). Spatiotemporal parameters were 
averaged across all steps for each participant. Time series of the kinematic data including gait events (e.g. heel strike) were exported as .csv files. Mean curves for ankle, knee and hip angles were computed for each leg and each participant using an in-house algorithm written in Matlab (MathWorks Inc., Natick, MA, USA) and used for further analyses

\subsection{Statistical analysis}

The required number of subjects was calculated based on group differences reported in the literature [1]. Sample size estimation revealed that 14 participants were required to detect an expected difference in knee ROM with an effect size of at least 1.12 with $80 \%$ power at a 5\% significance level. Because it was expected that SPM is at least as sensitive in detecting differences between groups as 0 -dimensional methods, we aimed at enrolling 20 participants per group.

For the spatiotemporal parameters, we were mainly interested in the clinical relevance of group differences, and hence computed $95 \%$ confidence intervals of group differences. In addition, independent sample t-tests were performed to detect statistically significant differences between groups. All tests were performed for self-selected walking speed for both groups and for self-selected walking speed of patients and matched walking speed of control subjects. The significance level was set a priori to 0.05 . All statistical analyses of spatiotemporal parameters were performed in SPSS Version 22 (IBM Corporation, Amonk, NY, USA).

For the kinematic parameters, we tested the null hypothesis that there are no differences in 1D kinematic data between patients with knee OA and asymptomatic control subjects when walking at self-selected normal speed and when walking at comparable speed. We examined the entire times series using SPM. All SPM analyses were conducted in Matlab (MathWorks Inc., Natick, MA, USA) using the open-source software package spm1D 0.4 (www.spm1d.org) [27]. Between-condition statistical analyses were conducted as described by Pataky [27]. 
Briefly, independent sample t-tests were performed for each walking speed to compare groups at normal and at comparable speed. The null hypothesis was rejected if the experimentally computed t-value for trajectory 1D data exceeded the critical value that was calculated based on Gaussian random field theory. The significance level for all statistical tests of the SPM analysis was set a priori to 0.05 . Additionally, mean differences in the joint angles were calculated by subtracting the mean of the control group from the mean of the patient group.

\section{Results}

\subsection{Clinical evaluation}

Eight patients had a K/L grade of 3, and 15 patients had a K/L grade of 4 . In patients with knee OA, the KOOS scores for the five sub-categories were (mean (SD)): KOOS Pain: 47 (13); KOOS Symptoms: 49 (17); KOOS ADL: 51 (19); KOOS Sport/Rec: 27 (21); and KOOS QOL 26 (17). In the control group the KOOS scores for the five sub-categories were: KOOS Pain: 98 (3); KOOS Symptoms: 99 (2); KOOS ADL: 99 (3); KOOS Sport/Rec: 98 (3); and KOOS QOL 98 (3). All KOOS scores were significantly lower in patients $(\mathrm{P}<0.001)$.

\subsection{Spatiotemporal parameters}

When walking at normal self-selected speed, all spatiotemporal parameters differed significantly between patients with knee OA and the control group (Table 1). The average normal self-selected walking speed in patients with knee OA was $0.4 \mathrm{~m} / \mathrm{s}$ slower than that of the control group $(\mathrm{P}<0.001)$. Furthermore, patients with knee OA had a longer stride duration $(+0.14 \mathrm{~s} ; \mathrm{P}<0.001)$, shorter stride length $(-0.22 \mathrm{~m} ; \mathrm{P}<0.001)$ and lower cadence $(-12.9$ steps $/$ min; $\mathrm{P}<0.001)$ than the control group. 
When being asked to walk at slow self-selected speed, the speed of the control group was comparable to that in patients with knee OA (Table 1). At this comparable speed, patients with knee OA had significantly shorter stride duration $(-0.09 \mathrm{~s} ; \mathrm{P}=0.027)$ and higher cadence ( +7.2 steps $/ \mathrm{min}, \mathrm{P}=0.020)$ but similar stride length compared to the control group.

\subsection{Kinematic parameters}

For walking at normal self-selected speed, SPM revealed that patients with knee OA had significantly lower knee flexion angles from the loading response to mid stance phase (4$24 \%$ of the gait cycle, maximum difference: $\left.-6.8^{\circ}, \mathrm{P}<0.001\right)$ and at the end of terminal stance to midswing phase $\left(60-77 \%\right.$ of the gait cycle, maximum difference: $\left.-11.0^{\circ}, \mathrm{P}=0.001\right)$ than the control group (Fig. 2). Furthermore, patients with knee OA had significantly greater ankle dorsiflexion and lower ankle plantarflexion, respectively from midstance to the initial swing phase $\left(8-68 \%\right.$ of the gait cycle, maximum difference: $\left.12.5^{\circ} ; \mathrm{P}<0.001\right)$ than the control group

(Fig. 2). At the hip joint, patients with knee OA had significantly lower extension during the terminal stance ( $38-54 \%$ of the gait cycle, maximum difference: $4.2^{\circ} ; \mathrm{P}=0.004$ ) than the control group (Fig. 2).

For walking at comparable speed, there were no more statistically significant differences between groups (Fig. 3). However, patients with knee OA still had smaller knee flexion during mid-swing phase than the control group (maximum difference: $-6.9^{\circ}$ ) (Fig. 3).

\section{Discussion}

In this study, we investigated the effect of walking speed on differences in spatiotemporal and kinematic parameters between patients with knee OA and asymptomatic control subjects by asking the control group to walk at normal and slow self-selected speed and 
detecting kinematic differences using SPM. We recruited only patients with severe knee OA who were already scheduled for a TKA as a homogenous patient group. Differences in spatiotemporal and kinematic parameters between patients with knee OA and the control group were present when participants walked at their normal self-selected speed but mostly disappeared when the control group walked at comparable speed to the patient group. These results have important clinical applications and must be considered when interpreting results of studies on gait in the context of knee OA.

Walking speed is known to influence spatiotemporal and kinematic parameters in healthy subjects [14]. However, the extent to which the known biomechanical alterations in patients with knee OA including changes in spatiotemporal and kinematic parameters are a result of reduced speed rather than disease progression remains unclear [15]. To clarify this question, research groups have performed gait analyses at different walking speeds [17, 28-31]. For instance, some groups encouraged patients (and control subjects) to walk faster or slower $[30,31]$ or at a predetermined speed on a walkway [29] or treadmill $[17,28]$. In our study, we chose to instruct the asymptomatic participant in the control group to walk at a slower selfselected speed rather than patients with knee OA to walk faster than their normal self-selected speed. It is possible that patients walk slower to minimize pain [32], and hence this factor may play a role when asking patients to walk faster than their normal self-selected speed. With our approach, we were able to elucidate which gait alterations detected at normal self-selected speed would remain significant at matched speed without asking patients with knee OA to walk at a non-preferred speed. Walking at a predefined faster than self-selected speed could lead to discomfort generating data that is not representative of habitual walking and difficulty or inability in performing the requested task. For instance, in the study by Bejek et al [28], only 14 of 20 patients completed the treadmill walking trial at $0.8 \mathrm{~m} / \mathrm{s}$ and only 11 the trial at 1.1 $\mathrm{m} / \mathrm{s}$. In our study, walking speed was comparable between patients with knee OA and the control group when the latter group was instructed to walk slower, hence allowing for 
comparison of spatiotemporal and kinematic parameters that are presumably caused by the disease rather than by differences in walking speed.

At normal self-selected speed, patients with knee OA had lower walking speed, higher stride duration, lower stride length and lower cadence compared to the control group. These results are in agreement with the literature on patients with knee OA. Authors reporting on severe knee OA have observed reduced speed and stride length in these patients $[5,6]$ and various studies including a systematic review have concluded that patients with severe knee OA have a longer stride duration and lower cadence $[1,7,8]$. At comparable speed, patients with knee OA had shorter stride duration, higher cadence, and a shorter, although not statistically significant, stride length compared to the control group. Similarly, Bejek et al. [28] reported higher cadence and shorter step length in patients than in control subjects at four different controlled speeds $(0.3$ to $1.1 \mathrm{~m} / \mathrm{s})$ on a treadmill. Moreover, Baliunas et al [31] instructed patients to walk slow, normal and fast and found shorter stride length in patients than in control subjects at matched speed conditions. Contrary to these findings, Liikavajo et al. [29] and Landry et al. [30] reported no difference in stride length.

Regarding joint kinematics, patients with knee OA had smaller peak knee flexion angles than the control subjects during midstance and early swing phase at normal self-selected speed. Moreover, ankle and hip angles differed between patients with knee OA and the control group during several phases of the gait cycle. Although comparing isolated values rather than the entire time series, Astephen et al. [5] came to the same conclusions for patients with severe knee OA. Moreover, Deluzio and Astephen [33] reported similar differences in knee kinematics between patients with knee OA and a control group throughout the gait cycle using principal component analysis. Interestingly, at comparable speeds, we found that all kinematic parameters no longer differed statistically significant between patients with knee OA and the control group. Zeni et al. [17] asked patients to walk at a self-selected speed and a predetermined speed of $1.0 \mathrm{~m} / \mathrm{s}$ and reported that the knee flexion ROM remained significantly 
between patients with knee OA and control subjects at $1.0 \mathrm{~m} / \mathrm{s}$. They concluded that apart from the knee flexion ROM all other biomechanical differences appear to be the result of altered speed. Moreover, Baliunas et al. [31] found the knee flexion ROM to be the sole difference under comparable speed. Landry et al. [30] did not find any kinematic difference at all. Mündermann et al. [34] found that only knee flexion at heel strike differed between patients with severe knee OA and age-matched controls but the average difference of $-3.7^{\circ}$ was below the boundary of clinical relevance $\left(5^{\circ}\right)$. Contrary to all other studies, Bejek et al. [28] detected significant kinematic differences regarding ROM of knee and hip flexion at each speed tested ( 0.3 to $1.1 \mathrm{~m} / \mathrm{s}$ ). However, only a part of the patient group was able to perform the trials in all four different speeds tested. Concluding, our results are mainly in accordance with the findings of the few previous attempts to measure kinematics at matched speeds. Furthermore, the present study increases the confidence in these findings, since, contrary to the previous studies, we proofed the kinematic differences for the entire gait cycle rather than comparing only discrete parameters.

Summarizing, this study adds to the attempts to clarify which gait alterations in patients with knee OA exist only as a consequence of reduced walking speed. While significant spatiotemporal differences in stride duration and cadence still exist, there were no significant differences in sagittal ankle, knee and hip kinematics at a matched speed.

Strengths and Limitations: To the best of our knowledge, this is the first study to combine inertial sensor based gait analysis and SPM to detect differences in spatiotemporal and kinematic parameters between patients with knee OA and asymptomatic control subjects at normal self-selected and comparable walking speeds. The strengths of this study are the inclusion of age-matched control subjects and the ability to capture measurements at a matched speed without encouraging patients to walk in an unnatural and potentially painful way. Although the involved cohort was rather small, this study can be considered a proof of principle 
study. In our statistical approach, we did not adjust for multiple comparisons (joints, groups). While this might increase the chance of finding significant differences, our results are in agreement with previous literature. Moreover, maximum differences in knee joint angles at selfselected speed were up to $6.8^{\circ}$ and $11.0^{\circ}$ which indicates that they are large enough to also be clinically and functionally relevant. The number of the subjects included in this study was based on the literature on discrete gait parameters. Moreover, patients involved in this study all suffered from severe OA and hence this patient group was rather homogeneous.

\section{Conclusion}

This study showed that most known kinematic alterations in patients with knee OA are dependent on the walking speed of the control subjects and that they disappear when control subjects walk at a comparable speed to the patients. This result highlights the importance of performing gait analysis at different and if possible matched speeds by letting the control group walk at several speeds. Moreover, this study provides evidence that inertial sensor gait analysis systems are suitable for detecting disease specific characteristics in ambulatory mechanics that are relevant in the context of diagnosis and treatment of knee OA.

\section{Conflict of interest statement}

The authors declare no conflict of interest.

\section{References}

[1] K. Mills, M.A. Hunt, R. Ferber, Biomechanical deviations during level walking associated with knee osteoarthritis: a systematic review and meta-analysis, Arthritis Care Res (Hoboken) 65(10) (2013) 1643-65. http://www.ncbi.nlm.nih.gov/pubmed/23554153. 
[2] I. McCarthy, D. Hodgins, A. Mor, A. Elbaz, G. Segal, Analysis of knee flexion characteristics and how they alter with the onset of knee osteoarthritis: a case control study, BMC Musculoskelet Disord 14 (2013) 169.

[3] J. Rahman, Q. Tang, M. Monda, J. Miles, I. McCarthy, Gait assessment as a functional outcome measure in total knee arthroplasty: a cross-sectional study, BMC Musculoskelet Disord 16 (2015) 66.

[4] S. Tadano, R. Takeda, K. Sasaki, T. Fujisawa, H. Tohyama, Gait characterization for osteoarthritis patients using wearable gait sensors (H-Gait systems), J Biomech 49(5) (2016) 684-690.

[5] J.L. Astephen, K.J. Deluzio, G.E. Caldwell, M.J. Dunbar, Biomechanical changes at the hip, knee, and ankle joints during gait are associated with knee osteoarthritis severity, $\mathrm{J}$ Orthop Res 26(3) (2008) 332-41.

[6] J. Weidow, R. Tranberg, T. Saari, J. Karrholm, Hip and knee joint rotations differ between patients with medial and lateral knee osteoarthritis: gait analysis of 30 patients and 15 controls, J Orthop Res 24(9) (2006) 1890-9.

[7] J.A. Zeni, Jr., J.S. Higginson, Dynamic knee joint stiffness in subjects with a progressive increase in severity of knee osteoarthritis, Clin Biomech (Bristol, Avon) 24(4) (2009) $366-71$.

[8] C.P. Chen, M.J. Chen, Y.C. Pei, H.L. Lew, P.Y. Wong, S.F. Tang, Sagittal plane loading response during gait in different age groups and in people with knee osteoarthritis, Am J Phys Med Rehabil 82(4) (2003) 307-12.

[9] S.C. Huang, I.P. Wei, H.L. Chien, T.M. Wang, Y.H. Liu, H.L. Chen, et al., Effects of severity of degeneration on gait patterns in patients with medial knee osteoarthritis, Med Eng Phys 30(8) (2008) 997-1003. 
[10] J.D. Childs, P.J. Sparto, G.K. Fitzgerald, M. Bizzini, J.J. Irrgang, Alterations in lower extremity movement and muscle activation patterns in individuals with knee osteoarthritis, Clin Biomech (Bristol, Avon) 19(1) (2004) 44-9.

[11] T.L. Heiden, D.G. Lloyd, T.R. Ackland, Knee joint kinematics, kinetics and muscle cocontraction in knee osteoarthritis patient gait, Clin Biomech (Bristol, Avon) 24(10) (2009) 833-41.

[12] J. Manetta, L.H. Franz, C. Moon, K.L. Perell, M. Fang, Comparison of hip and knee muscle moments in subjects with and without knee pain, Gait Posture 16(3) (2002) 24954.

[13] C.L. Hubley-Kozey, K.J. Deluzio, S.C. Landry, J.S. McNutt, W.D. Stanish, Neuromuscular alterations during walking in persons with moderate knee osteoarthritis, J Electromyogr Kinesiol 16(4) (2006) 365-78.

[14] C.A. Fukuchi, R.K. Fukuchi, M. Duarte, Effects of walking speed on gait biomechanics in healthy participants: a systematic review and meta-analysis, Syst Rev 8(1) (2019) 153.

[15] J.L. Astephen Wilson, Challenges in dealing with walking speed in knee osteoarthritis gait analyses, Clin Biomech (Bristol, Avon) 27(3) (2012) 210-2.

[16] M.D. Lewek, K.S. Rudolph, L. Snyder-Mackler, Control of frontal plane knee laxity during gait in patients with medial compartment knee osteoarthritis, Osteoarthritis Cartilage 12(9) (2004) 745-51.

[17] J.A. Zeni, Jr., J.S. Higginson, Differences in gait parameters between healthy subjects and persons with moderate and severe knee osteoarthritis: a result of altered walking speed?, Clin Biomech (Bristol, Avon) 24(4) (2009) 372-8.

[18] R. van der Straaten, L. De Baets, I. Jonkers, A. Timmermans, Mobile assessment of the lower limb kinematics in healthy persons and in persons with degenerative knee disorders: A systematic review, Gait \& Posture 59 (2018) 229-241. 
[19] C. Nuesch, E. Roos, G. Pagenstert, A. Mundermann, Measuring joint kinematics of treadmill walking and running: Comparison between an inertial sensor based system and a camera-based system, J Biomech 57 (2017) 32-38.

http://www.ncbi.nlm.nih.gov/pubmed/28366438.

[20] L. Donath, O. Faude, E. Lichtenstein, C. Nuesch, A. Mundermann, Validity and reliability of a portable gait analysis system for measuring spatiotemporal gait characteristics: comparison to an instrumented treadmill, J Neuroeng Rehabil 13 (2016) 6.

[21] G. Bailey, Assessment of Foot Kinematics During Steady State Running Using a Footmounted IMU, Procedia Engineering 72 (2014).

[22] T.C. Pataky, M.A. Robinson, J. Vanrenterghem, R. Savage, K.T. Bates, R.H. Crompton, Vector field statistics for objective center-of-pressure trajectory analysis during gait, with evidence of scalar sensitivity to small coordinate system rotations, Gait Posture 40(1) (2014) 255-8.

[23] C. Nuesch, E. Roos, C. Egloff, G. Pagenstert, A. Mundermann, The effect of different running shoes on treadmill running mechanics and muscle activity assessed using statistical parametric mapping (SPM), Gait Posture 69 (2019) 1-7. https://www.ncbi.nlm.nih.gov/pubmed/30658310.

[24] G. Sole, T. Pataky, E. Tengman, C. Hager, Analysis of three-dimensional knee kinematics during stair descent two decades post-ACL rupture - Data revisited using statistical parametric mapping, J Electromyogr Kinesiol 32 (2017) 44-50.

[25] L.J. Kellgren JH, The Epidemiology of Chronic Rheumatism: Atlas of Standard Radiographs of Arthritis, Blackwell Scientific Publications 1963.

[26] E.M. Roos, H.P. Roos, L.S. Lohmander, C. Ekdahl, B.D. Beynnon, Knee Injury and Osteoarthritis Outcome Score (KOOS)--development of a self-administered outcome measure, J Orthop Sports Phys Ther 28(2) (1998) 88-96. 
[27] T.C. Pataky, One-dimensional statistical parametric mapping in Python, Comput Methods Biomech Biomed Engin 15(3) (2012) 295-301.

[28] Z. Bejek, R. Paroczai, A. Illyes, R.M. Kiss, The influence of walking speed on gait parameters in healthy people and in patients with osteoarthritis, Knee Surg Sports Traumatol Arthrosc 14(7) (2006) 612-22.

[29] T. Liikavainio, T. Bragge, M. Hakkarainen, P.A. Karjalainen, J.P. Arokoski, Gait and muscle activation changes in men with knee osteoarthritis, Knee 17(1) (2010) 69-76.

[30] S.C. Landry, K.A. McKean, C.L. Hubley-Kozey, W.D. Stanish, K.J. Deluzio, Knee biomechanics of moderate OA patients measured during gait at a self-selected and fast walking speed, J Biomech 40(8) (2007) 1754-61.

[31] A.J. Baliunas, D.E. Hurwitz, A.B. Ryals, A. Karrar, J.P. Case, J.A. Block, et al., Increased knee joint loads during walking are present in subjects with knee osteoarthritis, Osteoarthritis Cartilage 10(7) (2002) 573-9.

[32] A. Mündermann, C.O. Dyrby, D.E. Hurwitz, L. Sharma, T.P. Andriacchi, Potential strategies to reduce medial compartment loading in patients with knee osteoarthritis of varying severity: reduced walking speed, Arthritis Rheum 50(4) (2004) 1172-8. http://www.ncbi.nlm.nih.gov/pubmed/15077299.

[33] K.J. Deluzio, J.L. Astephen, Biomechanical features of gait waveform data associated with knee osteoarthritis: an application of principal component analysis, Gait Posture 25(1) (2007) 86-93.

[34] A. Mundermann, C.O. Dyrby, T.P. Andriacchi, Secondary gait changes in patients with medial compartment knee osteoarthritis: increased load at the ankle, knee, and hip during walking, Arthritis Rheum 52(9) (2005) 2835-44.

http://www.ncbi.nlm.nih.gov/pubmed/16145666. 


\section{Figure captions}

Fig. 1. Lateral as well as posterior view of an individual wearing the RehaGait ${ }^{\circledR}$ inertial sensors used in the present study. There are 7 sensors, three placed at each leg, at the thigh, shank and foot and one placed posteriorly at the height of the 5th lumbar vertebra.

Fig. 2. Ankle (left), knee (middle) and hip (right) kinematics in patients with severe knee OA $(\mathrm{N}=23$; red) and asymptomatic control subjects $(\mathrm{N}=28$; black) for walking at normal selfselected speed. Top — mean and 1 standard deviation of joint angles normalized to one gait cycle; center - actual t-value (solid line) for independent t-tests determined using statistical parametric mapping and critical value (dashed line) based on Gaussian random field theory; bottom - difference between mean curves of patients with severe knee OA and the control group (positive values indicate larger values in patients).

Fig. 3. Ankle (left), knee (middle) and hip (right) kinematics in patients with severe knee OA $(\mathrm{N}=23$; red) and asymptomatic control subjects ( $\mathrm{N}=28$; black) for walking at comparable speed. Top — mean and 1 standard deviation joint angles normalized to one gait cycle; center-actual t-value (solid line) for independent t-tests determined using statistical parametric mapping and critical value (dashed line) based on Gaussian random field theroy; bottom-difference between mean curves of patients with severe knee OA and the control group (positive values indicate larger values in patients). 


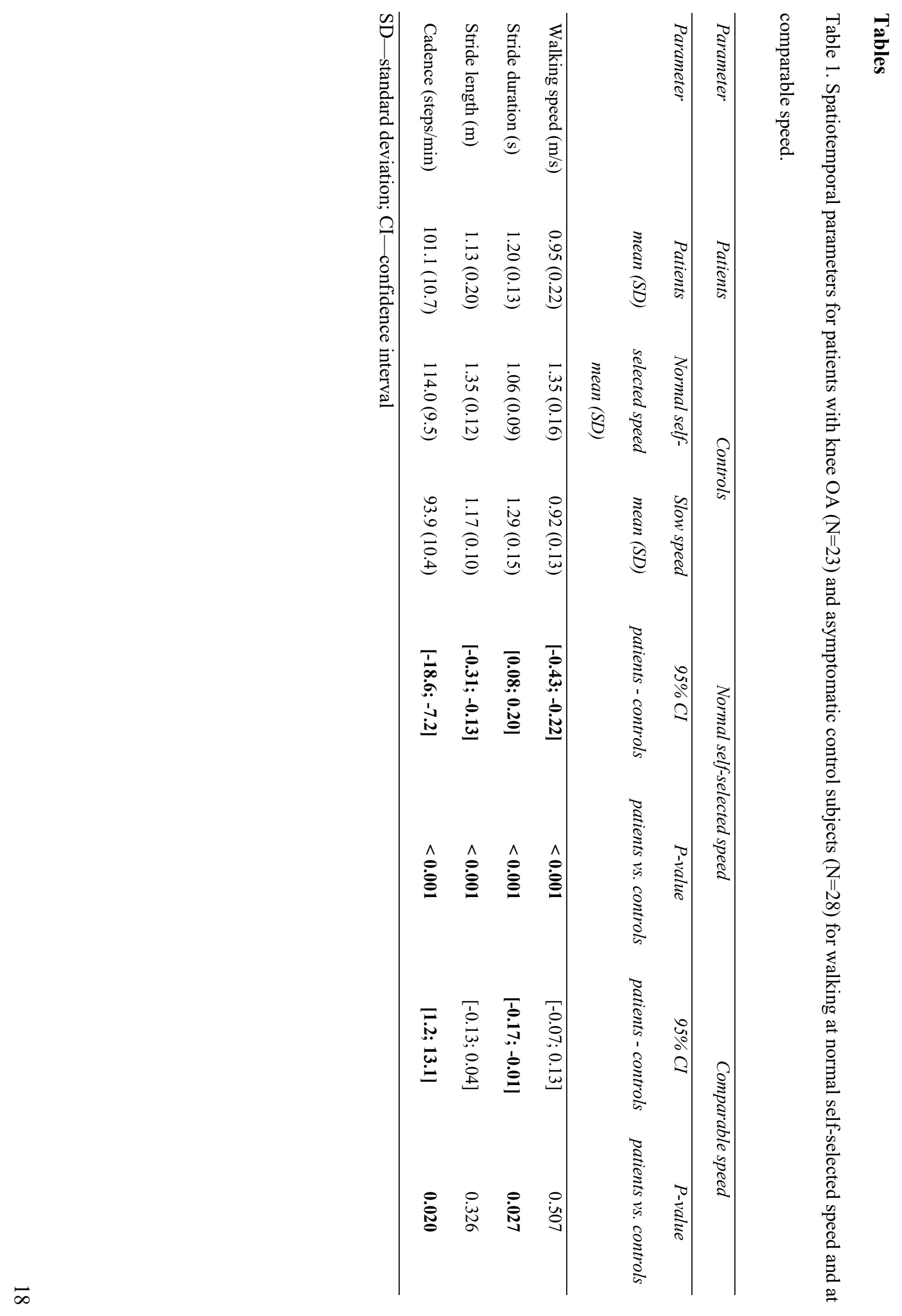



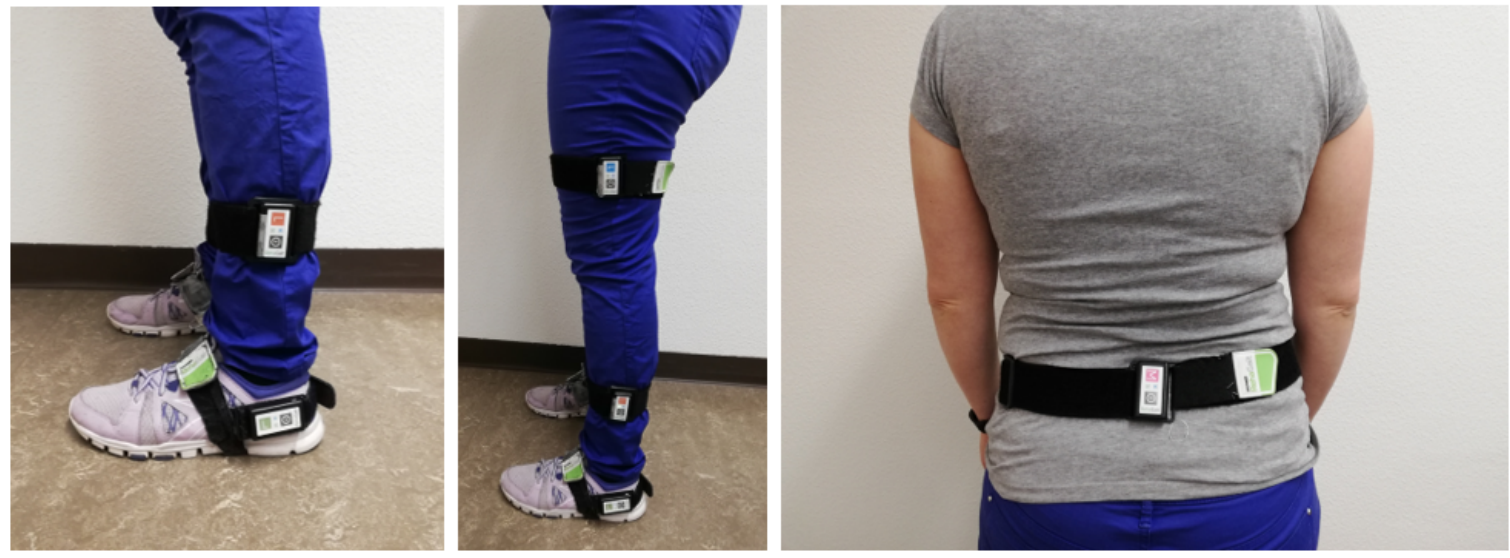

Figure 1 

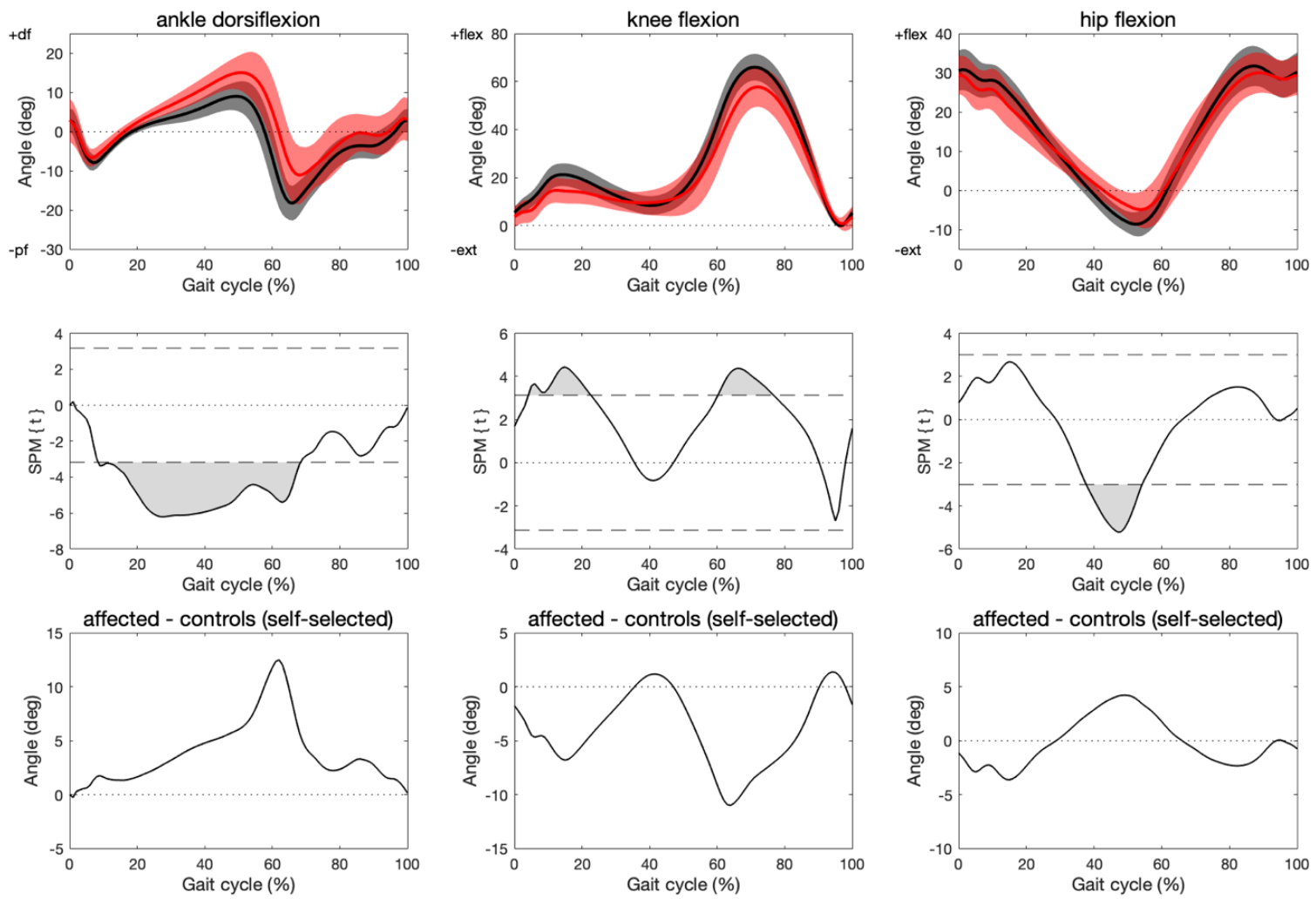

Figure 2 

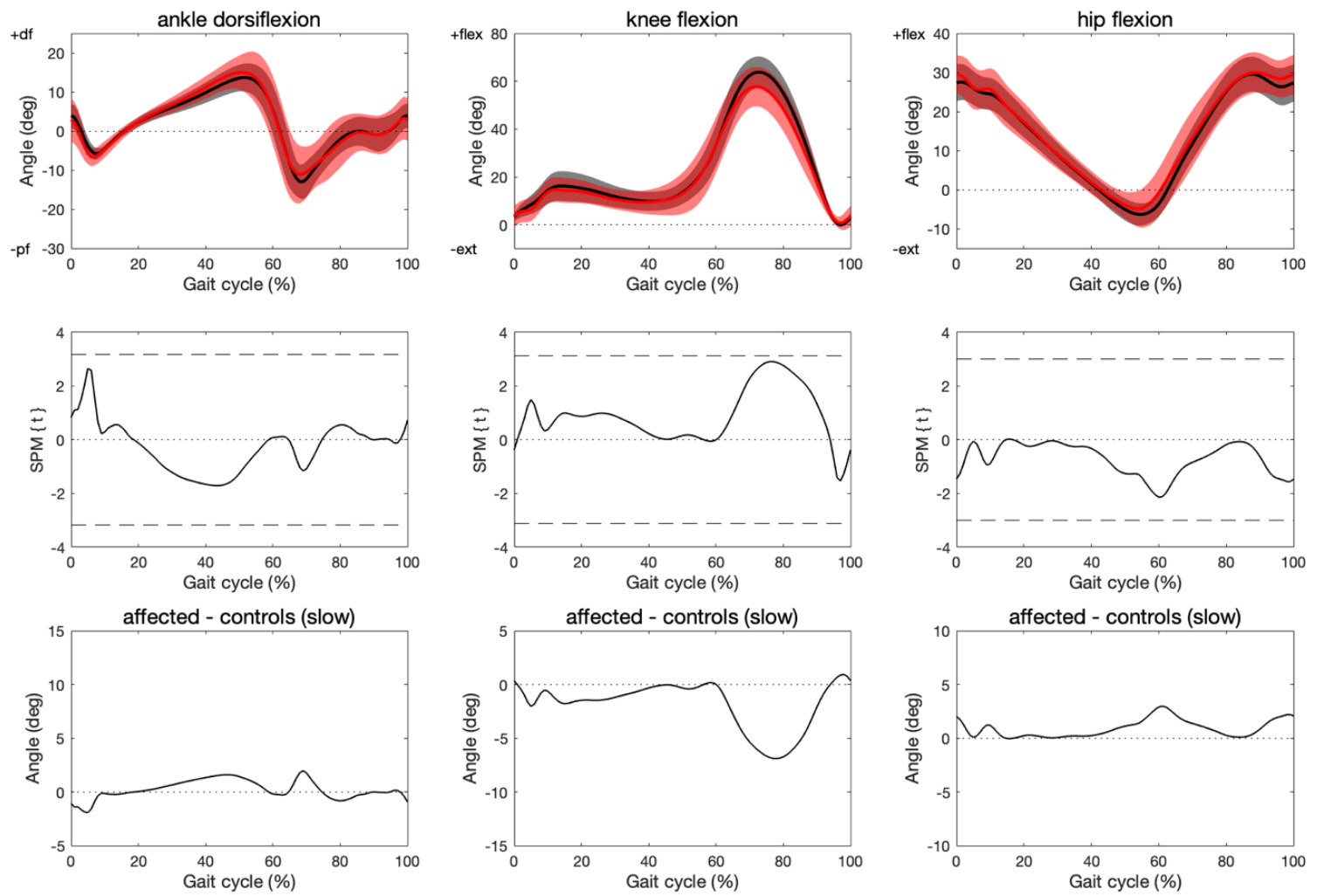

Figure 3 